\title{
Trivium
}

Revue franco-allemande de sciences humaines et sociales - Deutsch-französische Zeitschrift für Geistesund Sozialwissenschaften

31 | 2020

La culture politique de la République romaine

\section{Verlagerung der Funktionen und ideologischer Wandel: das Kapitol und das Forum des Augustus}

\section{Marianne Bonnefond-Coudry}

Traducteur : Andreas Wittenburg

\section{(2) OpenEdition}

\section{Journals}

Édition électronique

URL : https://journals.openedition.org/trivium/7161

DOI : 10.4000/trivium.7161

ISBN : 1963-1820

ISSN : 1963-1820

\section{Éditeur}

Les éditions de la Maison des sciences de l'Homme

\section{Référence électronique}

Marianne Bonnefond-Coudry, „Verlagerung der Funktionen und ideologischer Wandel: das Kapitol und das Forum des Augustus", Trivium [Online], 31 | 2020, online erschienen am 30 Juni 2020, abgerufen am 24 August 2021. URL: http://journals.openedition.org/trivium/7161 ; DOI: https://doi.org/10.4000/ trivium.7161

Ce document a été généré automatiquement le 24 août 2021.

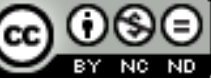

Les contenus des la revue Trivium sont mis à disposition selon les termes de la Licence Creative Commons Attribution - Pas d'Utilisation Commerciale - Pas de Modification 4.0 International. 


\title{
Verlagerung der Funktionen und ideologischer Wandel: das Kapitol und das Forum des Augustus
}

\author{
Marianne Bonnefond-Coudry \\ Traduction : Andreas Wittenburg
}

\section{NOTE DE L'ÉDITEUR}

\section{Originalausgabe | édition originale}

»Transferts de fonctions et mutations idéologiques, le Capitole et le Forum d'Auguste, dans L'Urbs«, Espace urbain et histoire, Rome, École française de Rome, 98, 1987, S. 251278.

Wir danken Frau Marianne Coudry für die freundliche Genehmigung, diesen Artikel in deutscher Übersetzung zu publizieren.

Nous remercions Mme Marianne Coudry de nous avoir accordé l'autorisation de traduire ce texte pour le présent numéro.

1 Unter allen von Augustus realisierten Bauten gelten der Tempel des Mars Ultor und das Forum, in das er eingegliedert ist, als die spektakulärsten und bezeichnendsten Beispiele einer neuen Konzeption der monumentalen Architektur, die in den Dienst der kaiserlichen Propaganda gestellt wird. Diese Funktion der politischen Legitimation, die von den modernen Forschern einstimmig unterstrichen wird, ist von Untersuchungen hervorgehoben worden, die sich im Wesentlichen mit der Architektur und der figürlichen Dekoration der Bauten beschäftigen. Sie haben dabei die beabsichtigte Bedeutung deutlich gemacht und gezeigt, wie sich in einer vollendeten Synthese die Themen des Sieges, der göttlichen Abstammung und des neuen Romulus miteinander vereinen, um das doppelte Erbe Caesars und der Republik zusammenzufügen. ${ }^{1}$

2 Man hat sich dagegen weniger für die Funktionen interessiert, die dem Tempel und dem Forum zugewiesen wurden und über die man doch hinreichend gut informiert ist, 
vor allem dank Cassius Dio und Sueton. Genauer gesagt hat man häufig nur jene Funktionen beachtet, die mit dem Krieg und der Feier des Sieges zu tun haben. Das sind die einzigen, die Sueton erwähnt, und sie entsprechen unmittelbar den Attributen des Mars. Unter den Themen, die das Monument dem Auge bietet, ist es dieser Bereich, den die Anwesenheit der triumphalen Quadriga im Zentrum des Platzes unterstreicht. ${ }^{2}$

Dagegen ist der Text des Cassius Dio in dieser Hinsicht von großem Interesse. Er bietet nämlich in sehr viel genauerer Weise als der Text des Sueton den wesentlichen Inhalt der lex templi, die zum Zeitpunkt der Weihung verkündet wurde. Und die Regelungen, die sie trifft, verdienen eine genauere Analyse, sei es auch nur aufgrund des Interesses, das Augustus selbst ihnen entgegenbrachte. Sueton gibt an, dass die Initiative auf ihn, Augustus, zurückgeht, und Cassius Dio bestätigt indirekt die Bedeutung, die dieser dem Gesetz beigemessen hat. Er stellt fest, dass Augustus die dedicatio selbst geleitet habe, und zwar in einer gewissen Abweichung von der Regel, denn er hatte diese Funktion ganz allgemein Caius und Lucius Caesar übertragen. Dio fügt sogar hinzu, dass Augustus das von ihm in jenem Jahr bekleidete Konsulat aufgegeben habe, und zwar noch vor dem Ende der Festivitäten, die die Weihung begleiteten, ${ }^{3}$ so als ob der Vollzug des Ritus der wesentliche Grund gewesen sei, aus dem heraus er die Magistratur bis zu jenem Datum beibehalten habe, und vielleicht habe er sogar deshalb entschieden, die Dedikation selbst zu leiten. ${ }^{4}$

4 Es geht uns hier darum, diesem Text zu seinem Recht zu verhelfen und die bisher vernachlässigten Passagen hervorzuheben. Auf der Grundlage des dann vollständigeren Bildes der dem Tempel und dem Forum zugesprochenen Funktionen soll die Bedeutung der von Augustus eingeführten Neuerungen bewertet werden, nicht allein in der Topographie der Stadt, sondern auch hinsichtlich der den öffentlichen Plätzen zugewiesenen Funktionen.

5 Wir wollen zunächst die beiden Texte vorstellen, die den Inhalt der lex templi angeben. Der wohlbekannte Text des Sueton findet sich in einer Passage der Biographie des Augustus, die sich mit den vom Kaiser errichteten öffentlichen Bauten beschäftigt und nacheinander das Forum und den Tempel des Mars Ultor aufzählt, den Tempel des Apollon auf dem Palatin und den Tempel des Iuppiter Tonans. Nachdem er ausgeführt hat, dass das Forum insbesondere dem Verfahren der Auslosung der Richter diente, fügt der Autor hinzu:

»Den Mars-Tempel hatte er gelobt, als er den Krieg von Philippi zur Rache für seinen Vater aufgenommen hatte; er ordnete daher an, dass sich der Senat hier über Kriege und Triumphe beraten und dass von dort denjenigen das Ehrengeleit gegeben werden sollte, die mit militärischem Kommando ausgestattet in ihre Provinzen aufbrachen, und dass die als Sieger Zurückkehrenden hier die Abzeichen ihrer Triumphe deponieren sollten. $\aleph^{5}$

Der Text des Cassius Dio steht im Buch 55. Durch eine Lücke im Manuskript haben wir den Anfang verloren, aber da die Aufzählung der Anordnungen trotz ihrer augenscheinlichen Zusammenhanglosigkeit einer bestimmten Logik folgt, kann man davon ausgehen, dass uns schlimmstenfalls einige Einzelheiten zu den Riten fehlen, die den Kaiser selbst betreffen. In der Übersetzung lautet der Text:

"dass er selbst und seine Nachkommen dorthin kommen sollten, sooft sie wünschten, und die, die aus dem Knabenalter herauswuchsen und unter den Epheben eingetragen wurden, sich ohne Ausnahme dorthin begaben; dass die, die zu den Kommandos außerhalb ausgesandt wurden, von dort aufbrechen sollten; dass der Senat dort die Beschlüsse über die Triumphe fassen sollte, und dass die 
Sieger dort dem Ares ihr Zepter und ihre Krone weihen sollten; und dass ihnen und allen anderen, die triumphale Ehren empfangen hatten, ihre Bronzestatue auf dem Forum errichtet werden sollte, wenn Feldzeichen in den Kriegen erobert und zurückgebracht wurden, sollten sie in diesem Tempel aufgestellt werden; und dass von den jeweiligen Führern der Ritterschaft neben seinen Stufen ein Fest gefeiert werden sollte; dass von denen, die den Zensus vollendet hatten, dort ein Nagel eingeschlagen werden sollte, und dass die Lieferung der im Pferderennen wetteifernden Pferde und die Bewachung des Tempels ebenfalls den Senatoren zukommen sollte, wie es für den Tempel des Apollon und des Iuppiter Capitolinus gesetzlich geregelt war. Mit diesen Bestimmungen weihte Augustus jenen Tempel, $[\ldots] . \ll^{6}$

\section{Die den Krieg betreffenden Bestimmungen}

7 Wir wollen zu Beginn die Vorschriften der lex templi betrachten, die den Krieg betreffen, weil sie die bekanntesten sind und scheinbar wenig Probleme bieten. Sie zeigen außerdem eine große Einheitlichkeit, denn die Praktiken, die sie einführen, betreffen allesamt die Vorbereitung und die Feier des siegreichen Kriegs.

Was die Sitzungen des Senats betrifft, so ist vorgesehen, dass die, welche dem Krieg und den Triumphen gewidmet sind, im Tempel abgehalten werden. Das war eine »besondere Funktion», die in der Zeit der Republik bereits für das Kapitol bestand, wie man bei Appian erfährt, der in Hinblick auf die Sitzung des Jahres 149 v. Chr., in der der Krieg gegen Karthago beschlossen wurde, schreibt: »Der Senat versammelte sich auf

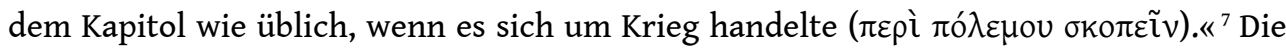
Angabe muss nicht so genau genommen werden, denn es gibt zahlreiche Fälle von Sitzungen dieser Art, die an anderen Orten abgehalten wurden, insbesondere in der Kurie. Aber vor allem bedeutet sie, dass das Kapitol ein mit starkem symbolischem Wert behafteter Ort war, wie es seine anderen Funktionen beweisen. Vor allem dort ist es, wo sich die religiösen und politischen Handlungen des ersten Tages eines Jahres vollzogen, dort ist es, wo die Texte der mit fremden Städten oder Völkern geschlossenen Verträge angeschlagen werden, dort ist es, wo die Triumphzüge enden. Wenn Augustus vorschreibt, dass die den Krieg betreffenden Sitzungen im Tempel des Mars Ultor abgehalten werden sollen, ist das nicht wirklich eine Neuerung. Er veranlasst lediglich eine Verlegung der Funktion weg vom Kapitol, das so eines Aspekts seiner Aufgaben beraubt wird. Dieser Vorgang der Enteignung geht im Übrigen sehr viel weiter und ist den heutigen Historikern keineswegs entgangen. ${ }^{8}$

Im Fall der die Triumphe betreffenden Sitzungen ist es ein wenig anders. In republikanischer Zeit konnten diese nicht an irgendeinem beliebigen Ort abgehalten werden, aber das aus Gründen, die in den an der Debatte teilnehmenden Personen begründet liegen und nichts mit den Gebäuden selbst zu tun haben. Da der Antrag für einen Triumph von den Feldherren selbst gestellt werden musste, die bei Überschreiten des pomerium ihr imperium verloren hätten, das notwendig war, um die Zeremonie zu feiern, mussten die Senatoren irgendwie zu ihnen kommen und sich außerhalb der Stadt versammeln. Es war also manchmal der Tempel des Apollon und manchmal der nahegelegene Tempel der Bellona, die die Versammlung beherbergten. Aber keiner dieser Tempel hatte vergleichbare Funktionen wie der des Kapitols, und nichts prädestinierte sie für diese Art von Versammlungen, wenn nicht Gründe praktischer Art. Der Umstand, dass diese Versammlungen sich ohne besonderen Grund in dem einen oder anderen Tempel abspielten, bestätigt diese Feststellung. Das lässt den 
innovativen Charakter der von Augustus erlassenen Regel für diese Sitzungen erkennen. Wenn Sueton schreibt, »er ordnete daher an«, stellt er damit klar, dass eine Verbindung zwischen dem Charakter des Tempels des Mars Ultor und der Entscheidung bestand, diese Sitzungen dorthin zu verlagern, und diese Verbindung ist offensichtlich die symbolische Bedeutung des Gebäudes. Das ist eine umso bemerkenswertere Neuerung, als sie einmalig erscheint. Nach unserer Kenntnis hat sich nichts anderes in Bezug auf die Orte der Versammlungen des Senats geändert. ${ }^{9}$

Die Verlegung der den Debatten über die Triumphe gewidmeten Sitzungen in den Tempel des Mars Ultor kann auch unter einem anderen Gesichtspunkt als gewagt erscheinen: Der Tempel liegt innerhalb der Grenzen des pomerium. Hätte Augustus sich über eine religiöse Tradition hinweggesetzt, die so stark war? In der Tat erlaubt ein Text des Sueton, der den einzigen Fall einer Sitzung im Tempel des Mars Ultor präsentiert, zu verstehen, wie sich der Respekt der Tradition und die Aufgabe der Praxis der republikanischen Zeit wohl miteinander in Einklang bringen ließen. Das ist die Passage im Leben des Caligula, die seinen Feldzug gegen die Germanen betrifft. Da er aus der freiwilligen Unterwerfung des Sohnes des Königs von Britannien, der von seinem Vater fortgejagt worden war, Ruhm für sich gewinnen wollte, »schickte der Kaiser einen großspurigen Bericht nach Rom. Seinen Kurieren trug er auf, auf einem Wagen direkt bis zum Forum und der Kurie zu fahren und den Konsuln das Schreiben $\mathrm{zu}$ überbringen, dies aber nur im Mars-Tempel und im Beisein des vollzählig versammelten Senats. ${ }^{10} \mathrm{Im}$ folgenden Text bestätigt sich, dass er von den Senatoren erwartete, dass sie einen Triumph für ihn beschlössen, und er veranlasste die Vorbereitungen in Gallien. Diese Beratungen fanden im Unterschied zu dem Verfahren in der Zeit der Republik in Abwesenheit des Betroffenen statt und es ist nicht mehr notwendig, die Sitzung außerhalb des pomerium abzuhalten. Diese Praxis geht auf die Zeit des Augustus zurück: die dem Tiberius gewährten Triumphe wurden ihm unter denselben Umständen übertragen.

11 Wir wollen zur lex templi zurückkommen. Eine weitere Bestimmung sieht vor, dass der Tempel des Mars der Ort des Aufbruchs derer sein solle, die Rom mit einem imperium versehen in Richtung ihrer Provinz verließen. Was bedeutet das genau? Weder Sueton noch Cassius Dio erwähnen ein besonderes Ritual, aber wenn man das mit der Zeremonie vergleicht, die sich bei dem Aufbruch eines Generals in der republikanischen Zeit vollzog, kann man die Absichten des Augustus erraten und ihre große Folgerichtigkeit feststellen. Die am meisten ins Einzelne gehenden Aufschlüsse findet man bei Livius. Sie betreffen die Abreise der Beamten, d.h. Konsuln und Prätoren, denn zu der Zeit, die der Bericht des Livius betrifft, waren Magistraturen und Promagistraturen noch nicht voneinander getrennt. Diese Beamten begaben sich, mit einem paludamentum angetan, auf das Kapitol, um vota zu geloben und die Auspizien für ihre Abreise einzuholen. Dann wurden sie von einem Gefolge von Bürgern bis zu den Mauern der Stadt begleitet und reisten direkt ab, um sich mit ihren Heeren zu vereinen. ${ }^{11}$ Man kann die Frage der Auspizien hier beiseitelassen. Abgesehen davon, dass sie nur selten erwähnt werden, hat der Umstand, dass sie später, als das imperium militiae getrennt von dem imperium domi bekleidet wurde, von den Promagistraten nicht mehr intra urbem eingeholt werden konnten, offenbar die Zeremonie der Abreise nicht verändert. ${ }^{12}$

12 Das Ziel der Zeremonie ist offensichtlich, den Schutz des Iuppiter Capitolinus für die Armee herbeizurufen. Das ist die Bedeutung der vota, und der Gott wird von dem 
siegreichen General bei seiner Rückkehr durch Geschenke geehrt. Diese Verbindung zwischen vota und dona kommt sehr deutlich in der berühmten Rede zum Ausdruck, die Livius dem Servilius in den Mund legt, der vor dem Volk die Bedeutung des Triumphs erläutert, um die Gegnerschaft derer zu entwaffnen, die ihn Aemilius Paullus verweigern wollten:

»Haben nicht eure Vorfahren jede große Unternehmung bei ihrem Anfang mit den Göttern begonnen, und sie bei der Beendigung ebenso auf die Götter zurückgeführt? Wenn ein Konsul oder Praetor im paludamentum mit seinen Liktoren auszieht, so spricht er auf dem Kapitol sein Gelübde aus. Wenn er als Sieger nach Beendigung des Krieges triumphierend auf dem Kapitol erscheint, führt er ebenfalls den Göttern, vor denen er die Gelübde tat, die gebührenden Dankgeschenke des Römischen Volks zu. «13

Wir erfahren bei Livius auch, dass die feierlichen Versprechen nicht allein an Iuppiter gerichtet sind, was ein anderer Text ausdrücklich feststellt, ${ }^{14}$ und dass die entsprechenden Geschenke bei Gelegenheit des Triumphs geweiht werden. Diese beiden Einzelheiten erlauben ein Verständnis der von Augustus erlassenen Regel, da man doch weiß, dass er ebenfalls vorschreibt, dass ein Teil der Feier des Triumphs sich im Tempel des Mars Ultor abspielen soll. Wenn die Generäle verpflichtet sind, aus dem Tempel den Ort ihres Aufbruchs zu machen, so geschieht das, weil sie dort ihre Gelübde ablegen und aus Mars Ultor einen der Beschützer ihrer Unternehmungen machen sollen.

In der Tat scheint es im Gegensatz zum Fall der Sitzungen des Senats nicht der Fall zu sein, dass das Kapitol seine vorhergehende Funktion als ein Ort des Aufbruchs verloren hat. Eine Passage im Panegyricus des Trajan zeigt, wie er vor seiner Abreise nach Germanien "nach dem Brauch das Kapitol besteigt $\ll{ }^{15}$ In einem Bereich, in dem die Tradition, anders als für die Beratungen des Senats, unumstößlich und zwingend war, hat Augustus vorgezogen, statt der Abschaffung die maßvollere und diskretere Methode der Verdoppelung anzuwenden und den Generälen, die die Stadt verließen, aufzuerlegen, ihr Gelübde sowohl auf dem Kapitol wie im Tempel des Mars Ultor abzulegen. Aber er machte damit aus dem Tempel des Mars Ultor die letzte Etappe vor der wirklichen Abreise, was ein indirekter Weg war, ihm einen faktischen Vorrang vor dem Kapitol einzuräumen.

Die Bestimmungen der lex templi in Hinblick auf den Triumph bilden natürlich ein Ganzes mit vorangehenden Artikeln, und aus diesem Grunde nennen Cassius Dio und Sueton sie zusammen, auch wenn die jeweils von ihnen benutzte Reihenfolge der Darstellung im Einzelnen eine andere ist. Sueton gibt an, dass der Triumphator die insignia triumphorum im Tempel des Mars Ultor niederlegen soll; Cassius Dio bemerkt dazu genauer, dass es sich um das Szepter und die Krone handele. In welchem Maße verändern diese Neuerungen den Ablauf der traditionellen Feier des Triumphs, und welche Beziehungen zwischen Mars Ultor und Iuppiter Optimus Maximus schaffen sie?

Wir wollen zunächst anmerken, dass die nach der Weihung des Tempels des Mars Ultor gefeierten Triumphe, für die die Texte topographische Angaben machen, alle auf dem Kapitol enden. Als Tiberius seinen Sieg über die Pannonier und die Dalmater feierte, stieg er von seinem Wagen, um vor Augustus niederzuknien, bevor er das Kapitol bestieg. Claudius hielt sich bei seinem Triumph über die Briten an die Tradition, berichtet Cassius Dio, und erklomm die Stufen zum Kapitol sogar auf Knien. Und die Triumphe des Vespasian und Titus enden ebenfalls dort. ${ }^{16}$ Leider ist keiner dieser Belege hinreichend genau, um eine Antwort auf die Frage nach dem Weg des 
Triumphzugs zu geben. Hat Augustus dem Triumphator einen Umweg über den Tempel des Mars Ultor vorgeschrieben, wie er es für den Zug bei der Abreise des Generals getan hatte, oder nicht?

Es ist die Frage der Insignien, die eine Antwort nahelegen wird. Die antiken Autoren sind einhelliger Meinung über ihre symbolische Bedeutung. Livius präsentiert den Triumphator als »Iovis Optimi Maximi ornatu decoratus«, Servius bezeichnet das Szepter und die tunica palmata als die »insignia Iovis $«{ }^{17}$ Die dem Triumphator von nun an auferlegte Verpflichtung, diesen ornatus, der ihn während der gesamten Dauer der Zeremonie mit Iuppiter gleichsetzt, im Tempel des Mars Ultor niederzulegen, wäre schwerlich zu verstehen, wenn dieser Akt sich in die eigentliche Feier des Triumphs eingliederte, denn er würde eine radikale Veränderung ihrer Bedeutung mit sich bringen. Keiner der Texte, die die Triumphe vor der Zeit des Augustus beschreiben, macht Angaben darüber, was mit den Insignien geschieht. Andererseits wird aber der Brauch aufrechterhalten, dass der Triumphator den während der ganzen Zeit des Triumphzugs von ihm in der rechten Hand gehaltenen Lorbeerzweig in gremio Iovis Optimi Maximi niederlegt. ${ }^{18}$ Man kann daher, wie es scheint, die von Augustus eingeführte Bestimmung in dem Sinne interpretieren, dass der Triumphator nach Abschluss des Triumphzugs die Zeichen des Triumphs dem Mars Ultor zum Geschenk machte. Man weiß, dass manche von diesen Zeichen von Männern, die mit einem Triumph geehrt worden waren, während der Spiele oder öffentlichen Feste wieder getragen werden konnten, und dass dieses Privileg sich auf die Kaiserzeit beschränkt. ${ }^{19}$ Es ist daher wahrscheinlich, dass Augustus, ohne den Ablauf des Triumphs zu verändern, von den Triumphatoren gefordert hat, dass sie sich am Ende des Triumphs in den Tempel des Mars Ultor begaben, um die Insignien der Obhut des Gottes anzuvertrauen, statt sie bei sich im Hause aufzubewahren. Das ist ein geschickter Weg, sie zu dem Bekenntnis zu verpflichten, dass sie ihm ihren Erfolg ebenso sehr verdanken wie Iuppiter, ohne dabei die Feier des Triumphs als solche in irgendeiner Weise zu ändern.

17 Wenn man sich vor Augen hält, dass von der Regierungszeit des Augustus an die einzigen militärischen Führer, die durch einen Triumph geehrt wurden, Mitglieder der kaiserlichen Familie waren, wird man außerdem in dieser Maßnahme den Ausdruck des dynastischen Charakters des neuen Regimes sehen.

Was hier von Interesse ist, ist die von Augustus angewandte Methode. Er wollte die Ideologie des Triumphs zum Nutzen des Regimes bewahren, das er dabei war zu errichten, und zugleich eine im politischen Leben der Republik so wesentliche religiöse Tradition respektieren. Ohne die rituellen Formen zu verändern und unter Ausnutzung der Lücken, die sie ihm boten, ist es ihm gelungen, einen Teil des Aufgabenbereichs des kapitolinischen Iuppiter auf Mars Ultor zu übertragen.

Die letzte Bestimmung der lex templi in Hinblick auf die Feier eines siegreichen Krieges schreibt vor, auf dem Forum des Augustus Statuen der Triumphatoren zu errichten, und ebenso derjenigen, die die Insignien eines Triumphs erhalten hatten. Eine Passage in den Annalen des Tacitus berichtet, wie Nero den Senat zusammengerufen habe (vermutlich im Tempel, denn der Text lautet "so als ob er die Taten des Krieges erläutern wolle«), um drei Männern die Ehren eines Triumphes zuzusprechen und ihnen Triumphstatuen »in foro « errichten zu lassen, d. h. ohne Frage auf dem Forum des Augustus. ${ }^{20}$ Diese Statuen sollten zu jenen bereits vorhandenen der summi viri der Republik hinzukommen, und in dieser Hinsicht wird das Forum des Augustus zum 
Rivalen und vielleicht sogar zum Erben der area Capitolina. ${ }^{21}$ Man weiß, dass letztere in der republikanischen Zeit von Statuen bevölkert war, die von Mitgliedern der nobilitas zu Ehren ihrer ruhmreichen Vorfahren errichtet worden waren, und die Augustus auf das Marsfeld hatte bringen lassen. ${ }^{22}$ Der von Sueton vorgebrachte Grund für diese Ortsveränderung - die Enge des Platzes - konnte sehr wohl einen anderen verbergen, nämlich den Wunsch, aus dem Forum den einzigen Platz im Zentrum der Stadt zu machen, der dem Ruhm der großen Männer der Vergangenheit gewidmet war, und durch die Monopolisierung dieser Funktion das Kapitol an den Rand zu drängen. ${ }^{23}$ Man wird dabei noch bemerken, dass auf dem Forum des Augustus auch Statuen der legendären Gründer und Vorfahren Roms standen. Aeneas sowie die Könige von Alba und Romulus stehen da wie ein Pendant zu den Götterstatuen, die in der area Capitolina aufgestellt sind. Doch die Präsenz einer vierten Gruppe von Statuen neben den drei genannten, die die direkten Vorfahren des Augustus umfasst, gibt dem Ganzen einen eindeutig ideologischen Zusammenhang und führt dem Beschauer die grundlegend monarchische Natur des augusteischen Staats konkret vor Augen. Es kommt noch ein bezeichnendes Detail hinzu: Die Männer, die in Zukunft die Ehrengaben eines Triumphs empfangen, werden nur auf dem Forum ein Zeugnis ihres Ruhms zurücklassen. Die Triumphatoren hingegen, d. h. die Mitglieder der kaiserlichen Familie, genießen das zusätzliche Privileg, die Insignien des Triumphs im Tempel selbst niederzulegen. Die neue politische Hierarchie tritt so an den Tag, und ihr entspricht eine sehr deutliche Hierarchisierung der Orte.

Ein Artikel der lex templi, den allein Cassius Dio erwähnt, sieht vor, dass die vom Feind wiedereroberten Feldzeichen im Tempel angebracht werden sollen. Diese Bestimmung zielt darauf ab, aus dem Anlass, der seine Errichtung gerechtfertigt hatte, nämlich die Rückführung der von den Parthern zurückgegebenen Feldzeichen, seine dauernde Berufung zu machen. Sehr aufschlussreich ist die "abwegige« Anwendung dieser Regel durch Caligula, nachdem er Lepidus hatte ermorden lassen. Er »weihte auch drei für seine Ermordung bestimmte Schwerter dem Mars Ultor«, schreibt Sueton, »und ließ eine erklärende Inschrift hinzufügen." Noch interessanter ist die Version des Cassius Dio: „Um seinen Tod zu feiern, verteilte er Geld an die Soldaten, als ob er Feinde besiegt hätte, und sandte dem Mars Ultor in Rom drei Schwerter. ${ }^{24}$ Die Schwerter werden mit den Feldzeichen gleichgesetzt und wie diese behandelt, denn die politische Unterdrückung wird wie ein Sieg über einen äußeren Feind behandelt. Die doppelte Eigenschaft des Mars Ultor als Garant des Sieges sowohl über den inneren Widerstand wie über den Feind von außen erlaubte eine solche Abweichung.

Auch in diesem Fall ist die Absicht offensichtlich: mit Iuppiter in einer seiner Funktionen wetteifern und ein Ritual, das die Unbesiegbarkeit Roms zum Ausdruck bringt, vom Kapitol in den Tempel des Mars zu verlagern.

Dieser ständige Wille, das Kapitol in seinen zur Glorifikation der siegreichen Kriege bestimmten Funktionen zu ersetzen, zeigt sich auch in punktuellen Initiativen wie der, die barbarischen Könige im Tempel des Mars Ultor ihren Eid schwören zu lassen, den Frieden und die von ihnen eingegangenen Verpflichtungen einzuhalten. Dieser Akt macht aus dem Gott den Garanten der Verträge und übertrug ihm eine der Aufgaben des Iuppiter, die durch die Publikation der Texte der »fast seit der Gründung der Stadt « geschlossenen Verträge auf dem Kapitol dokumentiert wurde..$^{25}$

Die Erfüllung einer gewissen Zahl von mit dem Krieg in Zusammenhang stehenden symbolischen Akten im Tempel des Mars Ultor, die vorher auf dem Kapitol vollzogen 
worden waren, zog auf diese Weise bisweilen eine Übertragung der Funktionen von Iuppiter auf Mars Ultor nach sich und dann wieder ihre Teilung zwischen den beiden Gottheiten. Die Folge war ein verhältnismäßiges Zurücktreten des Platzes des ersteren in diesem speziellen Bereich des bürgerlichen Lebens. Aber man wird bei Prüfung der übrigen Artikel der lex templi feststellen, dass die Aufgaben des Gebäudes von noch größerer Tragweite sind, und dass sich dabei ebenfalls, aber in weniger auffälliger Weise, eine Konkurrenz mit dem Kapitol zeigt.

\section{Die weiteren Funktionen des Tempels}

Alle Bestimmungen, die wir bisher untersucht haben und die ein zusammenhängendes Ganzes bilden, sind eine nach der anderen in dem Text des Cassius in einer Reihenfolge zusammengestellt, die dem logischen Ablauf der Ereignisse entspricht: Aufbruch zu einem Feldzug, Bewertung des Krieges und Beratung über den Triumph, Feier des Sieges und späteres Gedenken daran. Die Bestimmungen, die wir jetzt betrachten wollen, sind bis auf eine an das Ende des Berichts gesetzt. Und es ist insbesondere der Platz der Letzteren in der Aufzählung, die ihre Interpretation fraglich macht.

Zunächst zum Text: »dass er selbst und seine Nachkommen dorthin kommen sollten, sooft sie wünschten, und die, die aus dem Knabenalter herauswuchsen und unter den Epheben eingetragen wurden sich ohne Ausnahme dorthin begaben«. Wer sind »die, die aus dem Knabenalter herauswuchsen«? Man kann das dahingehend verstehen, dass es sich um unterschiedslos alle Söhne von Bürgern handelt. Das ist die Auffassung von Lily Ross Taylor, die entschieden die andere Vermutung zurückweist, nach der diese Maßnahme allein die Kinder der kaiserlichen Familie betreffe. ${ }^{26}$ Die Konstruktion des Satzes, die das Verbum an das Ende der beiden Sätze stellt und deren Subjekt grammatikalisch die beiden Gruppen sind, d. h. Augustus und seine Nachkommen einerseits und andererseits diejenigen, die aus der Kindheit herauswachsen, erlaubt indes eine restriktive Interpretation, und diese Frage kommt noch zu der nach der Reihenfolge der Darstellung der Bestimmungen der lex templi hinzu.

Man kann diese Einwände nicht so ohne weiteres beiseiteschieben, denn es ist die allgemeine Bedeutung der lex templi, um die es geht. Doch scheinen mir zwei Argumente Lily Ross Taylor recht zu geben. Zunächst ist da die Bedeutung des Ritus des Einschlagens des Nagels, die wir später erläutern werden, und dann die Gliederung am Ende des Texts, die Artikel zusammenfasst, deren Bestimmungen entweder die Gesamtheit der Bürgerschaft oder gewisse Teile davon betrifft, sie aber auf dem Wege über Handlungen oder Zeremonien in kollektiver Weise betrifft. Hier aber handelt es sich um einen individuellen Ritus, der dadurch mit anderen vergleichbar ist, die jedes Mitglied der kaiserlichen Familie aus eigenem Antrieb im Tempel vollziehen kann. ${ }^{27}$

Wir wollen also überlegen, welche Gruppe der jungen Bürger gemeint ist. Es ist vorgesehen, dass sie sich in den Tempel des Mars Ultor begeben, wenn sie aus dem "Knabenalter herauswuchsen«. Ohne Frage bezieht sich diese Disposition auf die Übernahme der toga virilis. Cassius verwendet in der Tat dieselben Worte, દiऽ toù

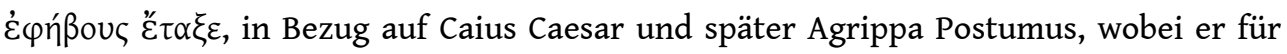
Ersteren ausführt, dass dieser zum gleichen Zeitpunkt das Recht erhält, an den Senatssitzungen teilzunehmen. Augustus selbst erwähnt dieses Ereignis selbst in den Rex Gestae mit folgenden Worten: "Sobald sie auf das Forum geführt wurden (es handelt sich um Caius und Lucius Caesar), beschloss der Senat, sie an den Versammlungen 
teilnehmen zu lassen « ${ }^{28}$ Wie man weiß, bezeichnet der Ausdruck in forum deducere im Allgemeinen die Zulassung des neuen Bürgers zu den öffentlichen Angelegenheiten zu dem Zeitpunkt, als er die toga virilis anlegte.

Inwiefern wird die traditionelle Zeremonie durch diese neue Bestimmung verändert? Die zahlreichen Texte, die sie uns überliefern, unterrichten uns, dass sie einen doppelten Charakter hatte. Im privaten Teil wurde die bulla und die toga praetexta den Laren dargebracht, und im öffentlichen Teil der Zeremonie begab sich der junge Mann auf das Kapitol, um dort Opfer darzubringen, in erster Linie der Iuventas, und wurde dann aufs Forum geführt, was ihn sofort in das bürgerliche Leben einband. Valerius Maximus berichtet von dem beispielhaften Fall des jungen Cotta, der »am selben Tag, als er die Toga virilis anlegte, sobald er vom Kapitol herabgestiegen war $"{ }_{,}^{29}$ aus Verpflichtung gegenüber seinem Vater einen Prozess gegen Carbo anstrengte. Sollte Augustus das Opfer auf dem Kapitol durch eines im Tempel des Mars Ultor ersetzt haben? In Wahrheit zeigt eine Passage bei Sueton, dass der Ritus auf dem Kapitol weiterhin bestand. Aufgrund seiner schwachen Gesundheit wurde der junge Claudius "an dem Tag, an dem er die Toga virilis anlegte, um Mitternacht ohne feierliche Zeremonie in einer Sänfte auf das Kapitol getragen «. ${ }^{30}$ Der Halt im Tempel des Mars hat sich also in die Zeremonie eingefügt, ohne dass das Kapitol seiner früheren Funktion beraubt wurde. Aber diese Neuerung stellt das augusteische Gebäude auf eine gleiche Ebene mit dem Kapitol und macht aus ihm ein neues Zentrum, und zwar nicht allein der mit dem Krieg verbundenen Handlungen und Riten, sondern auch umgreifend des Lebens der Bürger, da dort der Eintritt des neuen Bürgers in die Gemeinschaft sakralisiert wird.

Dennoch lässt die Formulierung des Texts noch Fragen offen: Was bedeutet diese Eintragung auf die Listen der jungen Männer? Es handelt sich sehr wahrscheinlich dabei um die tabulae iuniorum, jene Listen der Wehrtüchtigen, die von Livius und Polybios für das 3. Jahrhundert v. Chr. erwähnt werden. Sie wurden regelmäßig von den Zensoren kontrolliert und sicher jährlich auf den aktuellen Stand gebracht, ${ }^{31}$ und es ist gut möglich, dass diese Maßnahme zum Zeitpunkt der Liberalia vorgenommen wurde, wenn das Anlegen der Toga virilis stattfand. Das legt ein Text des Dionys von Halikarnass nahe, in dem der Historiker, der angibt, seine Informationen aus den Annales des L. Piso zu beziehen, erklärt, dass Servius Tullius die Zahl der Bewohner der Stadt kennen wollte und »von denen, die geboren wurden, von denen, die starben, und von denen, die man unter die Männer einschrieb«. Letztere werden später als die effektiv Wehrtüchtigen bezeichnet. Zu diesem Zweck machte er es zur Pflicht, für jede Geburt eine Münze im Schatz der Iuno Lucina zu hinterlegen, im Schatz der Venus Libitina für jeden Tod, und in dem der Iuventas für jeden Eintritt ins Erwachsenenalter.

Die historische Glaubwürdigkeit dieser Einzelheiten spielt hier keine Rolle. Aber sie lassen doch darauf schließen, dass zwischen der religiösen Zeremonie und der Eintragung auf der Liste der adsidui eine Verbindung besteht. Vom Standpunkt der Topographie her empfiehlt sich eine interessante Hypothese. Diese Listen wurden, da sie Teil der Archive der Zensoren waren, mit Sicherheit im atrium Libertatis aufbewahrt. Das Gebäude, das auf dem Sattel lag, der das Kapitol und den Quirinal verband, bevor die Arbeiten der trajanischen Zeit ihn einebneten, befand sich also in unmittelbarer Nähe des Augustusforums. ${ }^{32}$ Wenn man davon ausgeht, dass dieses Gebäude nach den Bauarbeiten des Asinius Pollio seine Funktion behalten hat, so könnte man annehmen, dass die jungen Männer sich zur gleichen Zeit wie in den Tempel des Mars Ultor eben 
dorthin begaben, um den Verwaltungsakt zu erledigen, der ihre Eingliederung in die Bürgerschaft offiziell machte. $\mathrm{Zu}$ den Gründen ideologischer Art, die den Ritus rechtfertigten, bei dem es sich mit Sicherheit um ein Opfer handelte, kamen noch Gründe topographischer Art hinzu.

31 Diese beiden Arten von Gründen rechtfertigen auch den Ritual des Nagels, das von der lex templi begründet wird: „Dass von denen, die den Zensus vollendet hatten, dort ein Nagel eingeschlagen werden sollte«. Dieses Ritual erinnert natürlich an den des clavus annalis im Tempel des Iuppiter Capitolinus, das Livius in einem wohlbekannten Text beschreibt. Im Zusammenhang mit der Ernennung eines dictator clavi figendi causa zur Unterbindung einer Pest im Jahre 363 v. Chr. versucht er das Geschehen zu berichten und beruft sich dabei vor allem auf die Untersuchungen des L. Cincius. Der Historiker bringt hier zwei Überlieferungen durcheinander, und zwar die des außerordentlichen Einschlagens eines Nagels im Falle der Pest oder von politischen und sozialen Unruhen einerseits, die expiatorische Wirkung hatte, und andererseits die des jährlichen Einschlagens des Nagels, die den Kalender betraf. ${ }^{33}$ Eine solche Verwechslung zeugt von dem Erstaunen der Zeitgenossen des Augustus über ein in Vergessenheit geratenes und unverständlich gewordenes Ritual und könnte erklären, wie Augustus es wiederaufleben lassen und in den Tempel des Mars Ultor verlegen konnte. ${ }^{34}$ Aber seine Absicht geht ohne Zweifel über die einfach erneuerte Inkraftsetzung einer sehr alten Tradition hinaus, die er zum Vorteil "seines« Tempels eingesetzt hat. Er hat ihr ohne Frage eine wirklich neue Bedeutung gegeben.

Das Ritual soll von denen vollzogen werden, "die den census abgehalten haben« (oi $\tau \iota \mu \eta \tau \varepsilon v ́ \sigma \alpha v \tau \varepsilon \varsigma)$, d. h. zum Zeitpunkt, als das lustrum abgeschlossen war. ${ }^{35}$ Dieser Abschluss bestand in einem suovetaurile, das von den Zensoren auf dem Marsfeld in Anwesenheit des neuen Heeres in Erfüllung eines von ihren Vorgängern ausgesprochenen Gelübdes vollbracht wurde. Gleichzeitig wurden neue Gelübde abgelegt, die den Schutz des Mars für das Volk der Bürger herbeiriefen und ein neues Opfer am Ende des nächsten census versprachen. Diese Zeremonie, die Valerius Maximus im Zusammenhang mit der Zensur des Scipio Aemilianus beschreibt, wird in der Zeit des Augustus auf dieselbe Weise vollzogen, wie ein Text des Sueton belegt. ${ }^{36}$ Ihre Bedeutung ähnelt der des Einschlagens des Nagels. Die beiden Rituale markieren den Übergang von einer Periode zur nächsten, und ein lustrum ist im chronologischen Sinne des Worts gemeint als Zeitraum, innerhalb dessen die Bürgerschaft entsprechend der von den Zensoren festgelegten Einteilung leben wird. Diese Riten gliedern die Zeit des bürgerlichen Lebens. Gleichzeitig »heiligen« sie den census, indem sie den Schutz des Mars herbeirufen.

33 Dazu kommt noch, dass das lustrum mit Augustus ein Monopol des Kaisers wird. Am Ende des letzten census seiner Regierungszeit ist Augustus durch ein Vorzeichen beunruhigt, dass er als Ankündigung seines baldigen Todes versteht. Er verzichtet darauf, die Gelübde zu leisten, und gibt seinem Kollegen Tiberius, wie Sueton berichtet, den Auftrag, das zu tun. Das ist ein Hinweis auf seine Auffassung, dass die Funktion des Zensors von Natur her dem Kaiser zukomme. Wie das Aussprechen der Gelübde ist auch das Einschlagen des Nagels mit Sicherheit ein dem Kaiser allein vorbehaltener Akt und ist dazu bestimmt zu zeigen, dass die Gemeinschaft der Bürger buchstäblich in seine Hände gelegt ist. Beziehung zwischen Mars Ultor, dem Kaiser und dem populus. Indem er die periodische 
Neugründung der Stadt, die der Zensus doch bedeutet, unter den Schutz einer dynastischen Gottheit stellt, macht Augustus einmal mehr aus Mars Ultor den Konkurrenten des Iuppiter Capitolinus, aber das geschieht in diesem Falle auf sehr viel grundlegendere Weise, denn die gesamte Bürgerschaft ist hier betroffen.

Es sei hier noch angemerkt, dass sich eine topographische Verbindung zwischen dem Tempel des Mars Ultor und den Zensoren ergibt. Da das Einschlagen des Nagels das Ende der Tätigkeit der Zensur markierte, musste sie sich zur gleichen Zeit vollziehen wie die Niederlegung der neuen Listen der Bürger im benachbarten atrium Libertatis.

Jeder Bürger unterhält auf diese Weise eine doppelte Beziehung zum Mars Ultor, und zwar individuell, wenn er die Toga Praetexta ablegt, und kollektiv, wenn der census ihm seinen Platz in der Hierarchie der Bürger zuweist. Genau diesen Aspekt heben die übrigen Bestimmungen der lex templi hervor: sie zielen darauf ab, auf dem Wege über Riten oder materielle Akte eine Beziehung zwischen dem Tempel und einem Teil der Bürgerschaft herzustellen, und dabei handelt es sich um die oberen Stände.

Zunächst ist das der Ritterstand: »dass von den jeweiligen Führern der Ritterschaft (oi

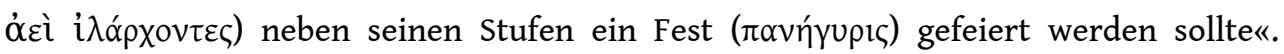
Wenn auch kein Zweifel daran bestehen kann, dass die Befehlshaber der Ritterschaft die seviri equitum sind, die infolge der von Augustus veranlassten Reorganisation an der Spitze der sechs turmae standen,${ }^{37}$ kann man die Art und Bedeutung dieses »Fests « nicht ebenso leicht verstehen. Wir wollen zu Anfang festhalten, dass der Zusammenhang uns, wie mir scheint, dazu zwingt, die seviri nicht als Einzelpersonen anzusehen, sondern in ihrer Eigenschaft als Repräsentanten und Sprecher der equites. Die von der lex templi vorgeschriebenen Handlungen und Riten gehorchen alle diesem Prinzip der Vermittlung. ${ }^{38}$ Außerdem legt der Terminus ( $\left.\pi \alpha v \eta ́ \gamma v \rho ı \varsigma\right)$, so ungenau er auch sei, eine Versammlung nahe. In welcher Situation können die in turmae zusammengefassten equites auf dem Forum des Augustus zu Füßen des Tempels versammelt werden? Lily Ross Taylor hat an die Ludi Martiales oder die Ludi Sevirales gedacht. ${ }^{39}$ Die erste Annahme ist wenig überzeugend, denn man sieht kaum ein, warum bei dieser Gelegenheit besondere Maßnahmen für die Ritter getroffen werden sollten, und im Übrigen bleiben die Quellen, die diese Spiele erwähnen, in dieser Hinsicht stumm. Die zweite Hypothese ist insofern naheliegender, als die Ludi Sevirales speziell die seviri equitum betrafen. Auch wenn ihre erste Erwähnung eineinhalb Jahrhunderte nach der lex templi liegt, ${ }^{40}$ könnte die Schaffung dieser Funktion doch in die augusteische Zeit zurückreichen und mit der Veränderung der Gliederungen des Ritterstands in Verbindung stehen.

Eine andere Interpretation wäre im Zusammenhang mit den Bestimmungen der lex templi noch einleuchtender, nämlich dass sich diese Zeremonie im Rahmen der transvectio equitum an den Iden des Juli vollzog. Weil diese Initiative seine Zeitgenossen besonders beeindruckt hat, weiß man, dass Augustus die in Vergessenheit geratene Tradition wiederbelebt hat, ${ }^{41}$ und dass bei gerade dieser Gelegenheit die Ritter in turmae gegliedert antraten. Mehr noch: er änderte den Charakter des Aufmarschs und fügte eine Inspektion hinzu, die der der Zensoren glich. ${ }^{42}$ Die Beziehungen zwischen der Funktion der Zensoren und dem Tempel des Mars Ultor stützen diese Auffassung ebenso wie der Umstand, dass die Funktion eines sevir equitum zunächst Caius und dann Lucius Caesar übertragen wurde, was den dynastischen Zusammenhang dieses Ritus hervorhebt.

39 In welcher Hinsicht beeinflusst diese Feier im Tempel des Mars Ultor die traditionelle der transvectio, wenn es tatsächlich bei dieser Gelegenheit ist, dass sie stattfindet? Einst 
nahm der Zug von dem an der Porta Capena gelegenen Tempel des Mars seinen Ausgang, überquerte das Marsfeld, wo im Tempel des Castor ein Opfer dargebracht wurde, und endete auf dem Kapitol. Das blieb, wie ein Text des Ulpian belegt, der Endpunkt. Der Tempel des Mars Ultor hatte also das Kapitol nicht ersetzt, aber die Strecke war vermutlich durch einen Umweg bereichert worden. ${ }^{43}$

Der letzte Artikel der lex templi, der den Senatorenstand betrifft, bietet keine Schwierigkeiten der Interpretation, auch wenn viele unbekannte Details bleiben. Er spricht den Senatoren das Recht zu, an den öffentlichen Ausschreibungen für die "Lieferung der im Pferderennen wetteifernden Pferde« und für die Aufsicht über den Tempel teilzunehmen. Es handelt sich mit Sicherheit um jene Versteigerungen, die während der Ludi Martiales stattfanden, denn andernfalls hätte Cassius Dio zweifelsohne genauere Angaben gemacht. Das Verfahren der locatio zur Finanzierung der öffentlichen Kulte ist auch sonst belegt ${ }^{44}$ und die Zuschläge können nur zu den Funktionen der Zensoren gehört haben. Dieser indirekte Hinweis zeigt deutlich, dass Letztere das hauptsächliche Thema am Ende der Erörterung des Cassius Dio sind.

41 Was den Umstand angeht, dass die Senatoren, die normalerweise von den publica ausgeschlossen bleiben, mitbieten dürfen, so zeigt er den Willen des Augustus, den Tempel des Mars Ultor zu einem Sonderfall zu machen und zu erlauben, dass sich zwischen ihm und den Senatoren ein persönlicheres Verhältnis etablierte als jenes, das sich aus der Einberufung des Senats bei den am Ende recht seltenen Gelegenheiten ergab.

Eine ähnliche Ausnahme gab es bereits, und zwar mindestens seit der Zeit Sullas, für die Lieferung der Viergespanne, die an den Rennen im Zirkus teilnahmen. ${ }^{45}$ Die lex templi erweitert lediglich den Anwendungsbereich, um den Euergetismus der Senatoren zu regulieren und zugleich einen Gewinn politischer Art daraus zu ziehen.

Eine letzte Bemerkung: Cassius Dio stellt klar, dass diese den Senatoren gewährte Ausnahme sich auch auf die Ausschreibungen für die Aufsicht über den Tempel des Apollon und des Kapitols bezogen. Der Tempel des Mars Ultor ist nicht der einzige, der in Konkurrenz zum Kapitol in seinem Anspruch als symbolisches Zentrum der Stadt steht. Von mancher Seite ist so die Rolle des Tempels des Apollon Palatinus bei dem Triumph des Jahres $29 \mathrm{v}$. Chr. hervorgehoben worden sowie die Überführung der Sybillinischen Bücher, die einst auf dem Kapitol aufbewahrt wurden. ${ }^{46}$ Jene Konkurrenz zwischen dem Kapitol und dem Tempel des Mars Ultor, deren verschiedene Aspekte wir vorangehend untersucht haben, ist Teil einer größeren Veränderung, die durch das allmähliche Zurücktreten des Kapitols zum Vorteil neuer Zentren gekennzeichnet ist, die in Verbindung mit dem sich einrichtenden Regime stehen.

Wenn man an diesem Punkt eine Bilanz der Lehren der lex templi aufstellen will, so muss man den Schluss ziehen, dass der Tempel des Mars Ultor und das Forum des Augustus nicht allein großartige repräsentative Bauwerke sind, die durch ihre architektonische wie ikonographische Botschaft die Macht Roms rühmen, die von ruhmreichen Vorfahren begründet worden war und durch den kaiserlichen Sieg garantiert wird. Sie sind auch ein neuer Ort der Bürger, wobei diese Bezeichnung einen metaphorischen Sinn annehmen muss, denn es vollzieht sich dort keinerlei Aktivität der Bürger im eigentlichen Sinne. Sie sind ein Ort, dessen Berufung durch die Vollendung von Handlungen und Riten definiert wird, die sich in zwei Kategorien unterteilen. Die einen feiern den siegreichen Krieg, die anderen den Zusammenhalt der sozialen Gemeinschaft, der regelmäßig bestätigt wird. Insofern einerseits der Triumph 
und andererseits die Zensur nunmehr ein Monopol des Kaisers sind, ist es die Besonderheit des neuen Regimes, die sich hier in gewisser Weise in die Topographie einschreibt. In diesem Sinne ist die Verlagerung gewisser Funktionen vom Kapitol in den Tempel des Mars Ultor oder die Einrichtung paralleler Funktionen von schwerwiegender Bedeutung: sie zeigt den Verfall des republikanischen Regimes an.

Doch man weiß, mit welchen Vorkehrungen und welcher Vorsicht diese politische Veränderung vollendet wurde. Dasselbe charakteristische Verhalten findet Anwendung bei der Entwicklung, die Mars an seinen Platz als Schutzgottheit des neuen Regimes geführt hat. Es ist durchaus von Nutzen, die Schritte dieses Vorgangs nachzuvollziehen und zu entdecken, wie die Rivalität zwischen Iuppiter Capitolinus und Mars Ultor sich in die Topographie der Stadt selbst eingeprägt hat.

\section{Chronologische Perspektive}

Diese Entwicklung vollzieht sich in der Zeit zwischen zwei Ereignissen: der Schlacht bei Philippi im Jahre $42 \mathrm{v}$. Chr., in deren Verlauf Octavius (der spätere Augustus) das Gelübde ablegt, dem Gott Mars einen Tempel zu errichten, und der Weihung dieses Tempels im Jahre $2 \mathrm{v}$. Chr. Aber um die Bedeutung besser zu erfassen, muss man auf die Vorgeschichte und Caesar zurückgreifen. Sueton nennt an erster Stelle der großen Vorhaben, die Caesar keine Zeit gehabt habe zu verwirklichen, »einen Mars-Tempel [...] von bislang unbekannter Ausdehnung ${ }^{47}{ }^{47}$ Das war ohne Frage ein Projekt, das mit dem Krieg gegen die Parther in Zusammenhang stand. ${ }^{48}$

Aber in einem anderen Zusammenhang erwähnt Appian, dass Caesar am Vorabend der Schlacht bei Pharsalus Mars und zugleich Venus angerufen habe, der er später den von ihm errichteten Tempel auf dem Forum geweiht habe..$^{49}$ Diese Anrufung »verwickelte« Mars also in den Bürgerkrieg, wie es bei dem Gelübde von Philippi von neuem der Fall war. Wenn das Projekt, diesen gewaltigen Tempel zu errichten, tatsächlich in Beziehung mit dem Feldzug gegen die Parther steht, kann man den Schluss ziehen, dass Mars bereits für Caesar mit dem Krieg verbunden ist, zugleich dem gegen den inneren Gegner wie dem gegen den äußeren Feind. Gerade in dieser doppelten Eigenschaft liegt das Besondere des Mars Ultor des Augustus.

Sueton sagt sogar noch etwas mehr über diesen Tempel des Mars, der niemals existieren sollte. Er solle errichtet werden, »wobei der See, auf dem er die Seeschlacht hatte aufführen lassen, aufgefüllt und eingeebnet würde». Es handelt sich da um die Naumachie, die bei Gelegenheit des Triumphs des Jahres $46 \mathrm{v}$. Chr. gestiftet wurde und die mehrere Autoren erwähnen, darunter vor allem Cassius Dio, der berichtet, wie der künstliche See auf dem Marsfeld angelegt wurde. ${ }^{50}$ Das ist ein wesentliches Detail für uns, denn es bedeutet, dass der Tempel, wie das für die dem Mars geweihten Tempel stets der Fall war, außerhalb des pomerium errichtet werden sollte.

Zwischen dem Gelübde bei Philippi und der Weihung des Tempels vergehen vierzig Jahre. Die Gründe für diese Verzögerung sind nicht allein materiell. Mars war "pro ultione paterna" angerufen worden, und Augustus vermeidet es während der Jahre des Aufbaus des neuen Regimes, die Erinnerung an Caesar und an den Bürgerkrieg zu erwecken, wie die Münzprägung dieser Zeit zeigt.

In den Jahren 20 bis $18 \mathrm{v}$. Chr., die als ein Wendepunkt in der von uns betrachteten Entwicklung erscheinen,${ }^{51}$ tritt Mars Ultor mit einer neuen Bedeutung wieder in den 
Vordergrund. Die von den Parthern zurückgegebenen Feldzeichen werden ihm geweiht, er wird so zum Rächer der Niederlagen der Römer und er erhält jene doppelte Berufung, die das »bis ulto« der Fasten des Ovid heraufbeschwören sollte. Dieser für uns ziemlich unerwartete Wechsel war es sicherlich weniger für die Zeitgenossen, insofern er sich wahrscheinlich an einem Vorbild Caesars inspirierte.

»Und in der Tat ordnete er an und veranlasste, Opfer dafür zu beschließen und einen Tempel für Mars Ultor auf dem Kapitol, in Nachahmung (לńn $\lambda \omega \mu \alpha)$ des Tempels für Iuppiter Feretrius, für die Weihung der Feldzeichen, und außerdem ritt er auf einem Pferd in die Stadt ein und wurde durch einen Triumphbogen geehrt. $\aleph^{52}$

Manche heutige Forscher haben die Existenz dieses Tempels in Zweifel gezogen, und die Frage bleibt umstritten. ${ }^{53}$

51 Cassius Dio präzisiert, dass der Tempel »in Nachahmung des Tempels des Iuppiter Feretrius « erbaut wurde. Die Aussage sollte nicht in konkretem Sinne verstanden werden. Der Tempel des Iuppiter Feretrius, der von Augustus wiederaufgebaut worden war, auch wenn man leider nicht weiß, zu welchem Zeitpunkt, wurde sicher unter Beachtung des alten quadratischen Plans wiederhergestellt, den Dionys von Halikarnass beschreibt, als er von seiner Gründung durch Romulus berichtet. Ein von Marcellinus $50 \mathrm{v}$. Chr. geprägter Denar bestätigt diesen Aspekt. ${ }^{54}$ Filippo Cassola gibt eine sehr viel zufriedenstellendere Interpretation des Texts, indem er zeigt, dass diesen beiden Tempeln gemeinsam war, ein adyton zu haben, da ihre Funktion ein und dieselbe war. Der eine sollte die Siegesbeute (spolia opima) bewahren, der andere die Feldzeichen. Das ist insofern gleichbedeutend, als in beiden Fällen die Beschränkungen des Zugangs zum Tempel sich aus dem Willen erklären, die von den Waffen ausgehende

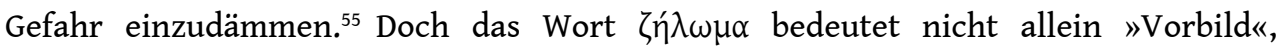
sondern hat auch die engere Bedeutung "Konkurrenz«, und es ist mit Sicherheit kein Zufall, dass Cassius Dio es verwendet. Mars Ultor als Bewahrer der von den Parthern zurückeroberten Feldzeichen befindet sich in der Tat durch die Errichtung seines Tempels auf derselben Stufe wie Iuppiter Feretrius. Es ist, als ob Augustus unter Ausnutzung einer neuen Situation - man kennt keinen anderen Fall von Feldzeichen, die vom Feind zurückerobert wurden - den Mars Ultor dazu einsetzt, den Iuppiter Feretrius zu »überholen«. Dessen Rolle beschränkt sich darauf, die Siegesbeute zu bewahren, die selten gewonnen wird, und das ist auch der Grund für die Vernachlässigung des Tempels vor seiner Wiederherstellung durch Augustus. Mars Ultor ist also noch nicht als Konkurrent des Iuppiter Capitolinus aufgeboten, denn sein Tempel ist zwar in gewisser Weise in den Einflussbereich des Gottes gestellt, aber doch nur als Rivale des Iuppiter Feretrius. In diesen Jahren zwischen 20 und $18 \mathrm{v}$. Chr. zeichnet sich also ein Vorgang ab, in dem, wie man sehen wird, Iuppiter Feretrius die Rolle eines Vermittlers spielt.

Es ist auch in diesem Zeitraum, dass die Grundlagen für die künftige Berufung des Mars Ultor gelegt werden. Die Funktionen, die vom Jahr $2 \mathrm{v}$. Chr. an die seinen werden sollten, sind von nun an vorgezeichnet. Das belegt der Text des Cassius Dio, der berichtet, dass Augustus bei Gelegenheit der Rückgabe der Feldzeichen eine ovatio feiert und durch einen Triumphbogen - den berühmten parthischen Triumphbogen geehrt wird. Und vor allem zeigen das die Themen, die von den zahlreichen zu dieser Gelegenheit geprägten Münzen vorgebracht werden, die in 17 Fällen den auf dem Kapitol errichteten Tempel darstellen.

53 Eine dieser Münzen stammt aus dem Atelier von Ephesus. Auf der Rückseite ist ein Rundtempel dargestellt, der ein Feldzeichen beherbergt und von der Legende MART 
ULTO begleitet ist. Die Vorderseite ist von entscheidendem Interesse, denn die Titulatur, die dort aufscheint, erlaubt es, die Prägung auf die Jahre 19 bis $18 \mathrm{v}$. Chr. zu datieren. ${ }^{56}$ Alle anderen Münzen sind in Spanien geprägt. Nur einige Einzelheiten auf der Rückseite erlauben es, die einen von den anderen zu unterscheiden. Die Legende fehlt bisweilen und im Inneren des Tempels sieht man in einigen Fällen eine Statue des Mars, der einen Adler und ein Feldzeichen hält, in anderen nur den Adler und die Feldzeichen und in noch anderen, was uns hier interessiert, den Adler und einen Triumphwagen. ${ }^{57}$ Die Bedeutung ist klar: Die Rückgabe der Feldzeichen wird mit einem Triumph gleichgesetzt, was noch weitere Münzen dieser spanischen Serien zeigen, die den parthischen Triumphbogen, die Quadriga und die Insignien des Triumphs zeigen. Auf diese Weise kommt der von diesem Zeitpunkt an hergestellte Zusammenhang zwischen dem Hinweis auf Mars Ultor und dem Ruhm des kaiserlichen Siegs sehr deutlich zum Ausdruck. Auf diesem Wege gelangt man dahin, dass Mars Ultor sich durchsetzen und Iuppiter einen großen Teil seiner Rolle und seiner traditionellen Funktionen entreißen sollte.

In dieser spanischen Serie gibt es auch eine Münze, die das Thema des Triumphs und die Titel eines parens und eines conservator miteinander verbindet. ${ }^{58}$ Sie ist von Andreas Alföldi kommentiert worden, der darin eine Vorausnahme der im Jahre $2 \mathrm{v}$. Chr. erfolgten offiziellen Zusammenstellung des Titels pater patriae sieht. Er zeigt, dass dessen schon im letzten Jahrhundert der Republik etablierte Bedeutung eine doppelte ist: Der Titel ehrt den, der die Stadt zugleich vor den äußeren wie den inneren Bedrohungen rettet. ${ }^{59}$ Diese spanische Münzprägung entwickelt also ein identisches Bild des Augustus und des Mars Ultor. Der Kaiser und der Gott finden sich da indirekt durch dieselbe doppelte Berufung vereint. Mehr als fünfzehn Jahre vor der Weihung des großen Tempels und des Forums sind die wesentlichen Züge ihrer ideologischen Botschaft festgelegt.

Eine Episode von anscheinend geringerer Bedeutung ereignet sich einige Jahre später im Jahre $8 \mathrm{v}$. Chr. und könnte einen Meilenstein in dieser Entwicklung darstellen, die den Vorrang des Mars Ultor besiegeln sollte. Bei seiner Rückkehr aus Gallien legt Augustus einen Lorbeerzweig im Tempel des Iuppiter Feretrius nieder, "gegen den Brauch«, wie Cassius Dio schreibt, ohne genauer zu erklären, warum es nicht Iuppiter Capitolinus ist, dem diese Ehre zuteilwird, wie es der von Augustus selbst im Jahre 29 v. Chr. respektierte Brauch war. ${ }^{60}$ Der Grund sind vielleicht die auf dem Kapitol im vorangehenden Jahr durch Unwetter angerichteten Schäden. ${ }^{61}$ Aber diese Wahl könnte auch den Wunsch zum Ausdruck bringen, dem Iuppiter Optimus Maximus das Monopol der Feier des Sieges streitig zu machen und einem Gott zu Ansehen zu verhelfen, der dem Mars Ultor schon nahestand und wenig später in seinen Umkreis treten sollte.

Wir wollen nun zum letzten Schritt des Jahres 2 v. Chr. kommen. Die Gleichzeitigkeit der Weihung des Tempels des Mars Ultor und der Einführung des Titels pater patriae ist bereits hinreichend hervorgehoben worden. Auf diese Weise wird endgültig die Verbindung hergestellt zwischen einerseits dem Gott, der die Bestrafung sowohl der Mörder Caesars sichert wie gleichfalls die, so fiktiv sie auch sei, der Sieger über Crassus, und andererseits der Berufung des Augustus als Beschützer der Stadt gegen die äußeren Kriege und die innere Subversion. patriae Augustus zu diesem Zeitpunkt offiziell verliehen wurde, aber dass er bereits vor 
diesem Beschluss so genannt wurde. ${ }^{62}$ Das ist ein klarer Beleg für die Kontinuität zwischen der Etappe der Jahre 20 bis $18 \mathrm{v}$. Chr. und der vorliegenden.

In den Res gestae gibt Augustus im Übrigen an, dass der Titel auf der Basis der im Zentrum des Forums aufgrund eines Senatsbeschlusses aufgestellten triumphalen Quadriga eingemeißelt sei. ${ }^{63}$ Auf diese Weise bestätigt sich die Rolle, die die rühmende Hervorhebung des kaiserlichen Sieges in der Festigung der Verbindung von Mars Ultor und Kaiser und damit im Zurücktreten des Iuppiter Capitolinus gespielt hat.

59 Er fügt noch hinzu, dass die Inschrift ebenfalls in seiner Wohnstätte eingemeißelt werden sollte. Das ist eine Maßnahme, die in Verbindung mit dem dynastischen Charakter des Regimes zu sehen ist, auf den mehrere Artikel der lex templi beharrlich hinweisen. Man findet im Übrigen noch eine weitere bezeichnende Übereinstimmung zwischen der Form, in der der Titel verliehen wurde, und den Auswirkungen der lex templi.

60 "Der Senat, der Ritterstand und das ganze Römische Volk haben mich Vater des Vaterlandes genannt«. Die Bestimmungen über das Anlegen der Toga virilis, das Einschlagen des Nagels, über die transvectio equitum und die Teilnahme der Senatoren am Zuschlag bestimmter Kosten des Kults sind wie ein Echo dieses Texts. Diese Riten, die sich an die Gottheit richten und dabei in der Beziehung zwischen dem Vater des Vaterlandes und der Gemeinschaft der Bürger vermitteln, werden deren Fortdauer sichern.

61 Am Ende wollen wir $\mathrm{zu}$ den topographischen Aspekten dieser letzten Etappen zurückkehren. Zunächst die Niederlegung der Feldzeichen: »Ich habe sie im adyton niedergelegt, das sich im Tempel des Mars Ultor befindet«, schreibt Augustus in den Res gestae, unmittelbar nach der Erwähnung seines diplomatischen Sieges über die Parther. ${ }^{64}$ Das ist ein Hinweis darauf, dass in seinen Augen die Tholos auf dem Kapitol nur ein vorläufiger Aufbewahrungsort war und ihre Errichtung nur eine Etappe im Prozess der Enteignung des Iuppiter.

62 Man weiß andererseits dank der Beschreibung Ovids, dass auf dem Forum des Augustus Romulus dargestellt war, wie er die sterblichen Überreste des Acro trug. ${ }^{65}$ Wie sollte man darin nicht ein Echo auf den Bericht sehen, den Dionys von Halikarnass wenige Jahre vorher verfasst hatte, um die Errichtung des Tempels des Iuppiter Feretrius durch Romulus zu erklären, der dort nach seinem Triumph die sterblichen Überreste des Acro weihte und so die Zuständigkeit des Gottes definierte? Der figürliche Schmuck des augusteischen Gebäudes zeigt auf, wie Iuppiter Feretrius auf dem Weg über das Thema Romulus geradezu in den Kreis um Mars Ultor hineingezogen wird und dem Kreis der Götter des Kapitols entgleitet. So vollendet sich eine Entwicklung, die sich seit langem abgezeichnet hatte und die indirekt zur Schwächung des Monopols des Iuppiter über das Gedenken an den Sieg beiträgt.

63 Und schließlich, und dieser Aspekt ist von entscheidender Bedeutung, findet sich Mars auf Initiative des Augustus innerhalb des pomerium angesiedelt, »in urbe « wie Ovid in aller Klarheit sagt, ${ }^{66}$ und zwar an einem Ort, der (anders als das Kapitol) durch nichts zu dieser Überschreitung vorausbestimmt war. Diese Verlagerung ist unmittelbarer Ausdruck der Erweiterung des Aufgabenbereichs des Gottes. Er ist nicht mehr auf den Schutz der Bürger unter Waffen beschränkt und wird auf einer Stufe mit Iuppiter eine Art Schutzgottheit der Stadt. Die Begründung der Ludi Martiales, die nach dem Vorbild 
der sehr alten dem Iuppiter gewidmeten Ludi Romani ausgerichtet werden, belegt das sehr deutlich. ${ }^{67}$

Wir wollen wiederholen und zusammenfassen: Augustus, der seiner Macht solide moralische Grundlagen verleihen wollte, hat zum Nachteil des Iuppiter, der eigentlichen nationalen Gottheit der Republik, einem Gott zur Geltung verholfen, der bis dahin auf eine zweitrangige Rolle beschränkt war und der als Vermittler in der Gleichsetzung von Staat und Kaiser dienen sollte. Die materielle Umsetzung, die diese Politik erfahren hat, ist von großem Interesse. Es handelt sich um eine Reihe von Initiativen zur Errichtung von Bauten, die dazu bestimmt waren, die Aufmerksamkeit auf einen neuen städtischen Bereich zu lenken und das Kapitol allmählich seiner Bedeutung zu berauben. Die Untersuchung dieses Prozesses hat gezeigt, mit welcher Umsicht diese Veränderung vorgenommen wurde. Zunächst wurde Mars Ultor auf dem Kapitol verankert und man schuf eine unklare Verbindung von gleichzeitiger Annäherung und Rivalität zwischen ihm und Iuppiter Feretrius. Das führte am Ende mit dem Umzug der beiden Gottheiten auf das neue Forum zur Verlagerung der dynamischsten Aspekte in der symbolischen Kraft des Sieges, deren Monopol Iuppiter einst innehatte.

\section{Abschließende Betrachtung}

Welche Einstellung hatte Augustus $\mathrm{zu}$ Iuppiter Capitolinus selbst? Die außerordentlichen Weihgaben, mit denen er ihn ehrte - sechzehntausend Pfund Gold, Edelsteine und Perlen im Wert von fünfzig Millionen Sesterzen, wie Sueton angibt ${ }^{68}$-, und der Wiederaufbau des Tempels, den er veranlasst, belegen eine sehr ausdrückliche Sorge der Ehrerbietung. »Ich habe das Kapitol [...] unter Aufwand großer Kosten wiederhergestellt, ohne jede Inschrift meines Namens «, schreibt er in den Res gestae. ${ }^{69}$ Diese Ehrerbietung ist gewollt distanziert und unpersönlich. Augustus beabsichtigt nicht, aus Iuppiter eine Gottheit zu machen, die dem neuen Regime nahesteht. Das belegt auch der Umstand, dass das Kapitol zwar bis auf wenige Ausnahmen seine alten Funktionen behält, aber keine neuen empfängt.

Was für einen Gegensatz bilden in dieser Hinsicht der Tempel des Mars Ultor und das Forum als wahre Pole der Erneuerung in den drei Bereichen der Architektur, der Ikonographie und der Funktionen! Mit Recht betrachtet man sie als das beste Beispiel der Nutzung an Orte gebundener Symbolik oder, anders gesagt, der materiellen Formgebung eines ideologischen Auftrags. Aber da ist noch mehr, und die Untersuchung der lex templi hat uns das zu verstehen gegeben. Ein Gebäude ist nicht allein dazu da, gesehen zu werden. Die Botschaft, deren Träger es ist, muss ständig durch den Vollzug regelmäßig wiederholter Handlungen und Rituale wiederbelebt und aktualisiert werden, die auf zwei grundlegende Aspekte der kaiserlichen Funktion hinweisen: die Führung der Kriege und die Kontrolle der Gemeinschaft der Bürger. Die Untersuchung der lex templi bestätigt und bereichert die visuelle Lektüre des Gebäudes. 


\section{BIBLIOGRAPHIE}

Alföldi, A. (1952): »Die Geburt der kaiserlichen Symbolik. Parens patriae«, MH, 9, S. 204-243.

Alföldi, A. (1953): »Die Geburt der kaiserlichen Symbolik. Parens patriae«, MH, 10, S. 103-124.

Alföldi, A. (1954): „Die Geburt der kaiserlichen Symbolik. Parens patriae», MH, 11, S. 133-169.

Béranger, J. (1953): Recherches sur l'aspect idéologique du Principat, Basel.

Bonnefond, M. (1979): »Le Sénat républicain dans l'atrium Libertatis?«, MEFRA, 91, S. 601-622.

Bonnefond-Coudry, M. (1989): Le Sénat de la République romaine, de la guerre d'Hannibal à Auguste.

Pratiques déliberatives et prise de décision, Rom.

Cassola, F. (1970): „Livio, il tempio di Giove Feretrio e la inaccessibilità dei santuari di Roma«, RSI, 81, S. 1-27.

Cassola, F. (1981): »I templi di Marte Ultore e i Ludi Martiales«, in: Scritti sul mondo antico in memoria di F. Grosso, Rom, S. 99-118.

Chioffi, L. (1996): Gli elogia augustei del foro romano. Aspetti epigrafici e topografici, Rom.

Demougin, S. (1988): L'ordre équestre sous les Julio-Claudiens, Rom.

Fears, J. R. (1981): »The cult of Jupiter and Roman imperial ideology«, in: ANRW, 11, 17, 1, S. 60-63.

Gagé, J. (1932): »Un thème de l'art impérial: la victoire d'Auguste«, MEFR, 49, S. 61-92.

Grandazzi, A. (2017): Urbs. Histoire de la ville de Rome des origines à Auguste, Paris.

Gros, P. (1976): Aurea templa. Recherches sur l'architecture religieuse de Rome à l'époque d'Auguste, Rom.

Heurgon, J. (1964): »L. Cincius et la loi du clavus annalis«, Athen, 42, S. 432-437.

Hurlet, F. (2010): »Recherches sur la profectio de la dictature de Sylla à la lex Pompeia (82-52). Le cas des gouverneurs de rang prétorien«, in: Barrandon, N. / Kirbihler, F. (Hg.): Administrer les provinces de la Rome républicaine, Rennes, S. 45-75.

Kaiserbiographien (2018): Caesar, Augustus, Tiberius, Caligula, Claudius, Nero, Galba, Otho, Vitellius, Vespasian, Titus, Domitian / C. Suetonius Tranquillus, hg. von U. Blank-Sangmeister, übers. v. Ursula Blank-Sangmeister, Marion Giebel, Hans Martinet und Dietmar Schmitz, Ditzingen.

Magdelain, A. (1968): Recherches sur l'imperium. La loi curiate et les auspices d'investiture, Paris.

Magdelain, A. (1978): La loi à Rome, histoire d'un concept, Paris.

Momigliano, A. (1930): »Ricerche sulle magistrature romane«, BCAR 58, S. 29-42.

Mommsen, T. (2017 [ $\left.\left.{ }^{3} 1887-1888\right]\right):$ Römisches Staatsrecht, Darmstadt.

Nicolet, C. (1966): L'Ordre équestre à l'époque républicaine (312-43 av. J.-C.), Bd. 1, Paris.

Nicolet, C. (1976): Le metier de citoyen dans la Rome républicaine, Paris.

Ogilvie, M. (1961): »Lustrum condere«, JRS, 51, S. 31-39.

Platner, S. B. /Ashby, T. (1929): A Topographical Dictionary of Ancient Rome, Oxford. 
Rich, J. (1998): »Augustus' Parthian honours, the temple of Mars Ultor and the arch of the Forum Romanum«, PBSR, 66, S. 71-128.

Sauron, G. (1994): Quis deum. L'expression plastique des idéologies politiques et religieuses à Rome à la fin de la République et au début du Principat, Rom.

Sauron, G. (2004): »Actualité du forum d'Auguste«, REL, 82, S. 25-31.

Simpson, C. (1993): »A Shrine of Mars Ultor revisited«, RBPh, 71, S. 116-122.

Spannagel, M. (1999): Exemplaria Principis. Untersuchungen zur Entstehung und Ausstattung des Augustusforums, Heidelberg.

Talbert, R. (1984): The Senate of Imperial Rome, Princeton.

Taylor, L. R. (1931): The Divinity of the Roman Emperor, Middleton.

Taylor, L. R. / Scott, T. (1969): „Seating space in the Roman Senate and the senatores pedarii«, TAPhA, 100.

Zanker, P. (1968): Forum Augustum, Das Bildprogramm, Tübingen.

Zanker, P. (1984): Il Foro di Augusto, Rom.

Zanker, P. (1987): Augustus und die Macht der Bilder, München, S. 196-217.

\section{NOTES}

1. $\mathrm{Zu}$ diesen Fragen hat es in den letzten fünfzig Jahren zahlreiche Untersuchungen gegeben. Die beachtenswertesten sind Zanker (1968), (1984), (1987), S. 196-217; Gros (1976); Sauron (1994), S. 525-536; Spannagel (1999), mit der Rezension von Sauron (2004).

2. So Taylor (1931), S. 200-202. Die Funktionen des monumentalen Komplexes werden in jüngeren Arbeiten besser zusammengefasst, vgl. z. B. Grandazzi (2017), S. 653-665.

3. Suet. Aug. 29,2; Cass. Dio 55,10,6.

4. Zu den charakteristischen Besonderheiten der lex templi s. Magdelain (1978), S. 29-31. Der Bericht des Cassius Dio zeigt, wie Augustus sich der Tradition anpasst, indem er einerseits als Magistrat handelt und andererseits die Weihung in Übereinstimmung mit den leges ausspricht, die gerade erst verkündet worden waren, vermutlich durch ihn selbst: Cass. Dio 55,10,5-6.

5. Suet. Aug. 29,2. [Übers. Ursula Blank-Sangmeister, Kaiserbiographien (2018)].

6. Cass. Dio 55,10,2-5.

7. App. Lib. 75. S. dazu Bonnefond-Coudry (1989), S. 69-79.

8. Taylor / Scott (1969), S. 560; Taylor (1931), S. 202; Fears (1981).

9. S. dazu Talbert (1984), S. 113-120.

10. Suet. Calig. 44,2 .

11. Vgl. dazu zuletzt Hurlet (2010).

12. S. Magdelain (1968), S. 51-57. Texte, die belegen, dass die Zeremonie am Ende der Republik und danach identisch blieb, sind: Cic. Verr. 2,5,34; Caesar Gall.1,6,6; Plin. Paneg. 5,2-3.

13. Liv. 45,39,11 (Übers. K. Heusinger).

14. Liv. $42,49,6$.

15. Plin. Paneg. 5,2-3.

16. Suet. Tib. 20,1: Cass. Dio 60,23,1; Ios. bell. Iud. 7,123-157.

17. Liv. 10,7,9-10; Iuv. 10,38; Serv. Ecl. 10,27.

18. Zu dieser Tradition Sil. 15,117-120; Pacatus Paneg.9,5. Und zur Fortdauer nach Augustus s. Plin. NH 15,133-134; Suet. Dom.6,2. 
19. S. Mommsen (2017), I, S. 438-440.

20. Tac. Ann. 15,72,1.

21. Diese Beobachtung hat bereits Fears (1981), S. 62, gemacht.

22. Suet. Calig. 34,2. Zu den Statuen, die die area Capitolina bevölkerten, s. Platner / Ashby (1929), S. 49.

23. Dieses Unternehmen war ungefähr zehn Jahre vorher durch die Aufstellung der Serie von tituli auf dem Forum Romanum in die Wege geleitet worden, und das Forum des Augustus nahm es nur in größerem Stile wieder auf (s. dazu Chioff [1996]).

24. Suet. Calig. 24,6; Cass. Dio 59,22,7.

25. Suet. Vesp. 8,5.

26. Taylor (1931), S. 201, Anm. 47.

27. So etwa der Kaiser Claudius, der dem Mars Ultor opfert, als ein Verschwörer festgenommen wird: Suet. Claud. 13,1.

28. Cass. Dio 55,22,4; 55,9,9 (Zon.); Mon. Anc. 14,1.

29. Vgl. die Texte, die in RE s.v. Toga und vor allem s.v. tirocinium fori zusammengestellt sind, vor allem Serv. 5,40; App. BC 4,30; Dion. Hal ant.4,15,5; Aug. Civ. 4,11; Val. Max. 5,4,4.

30. Suet. Claud. 2,5.

31. Liv. 24,18,7; Pol. 2,23,9. Zu ihrer jährlichen Überprüfung Mommsen (2017), II.1, S. 407-409; Nicolet (1976), S. 114.

32. Dion. Hal. ant. 4,15,5. Cf. Bonnefond (1979).

33. Liv. 7,3,5-8. S. dazu Heurgon (1964).

34. Momigliano (1930): »Dictator clavi figendi causa«.

35. Dazu bleibt die schon ältere Untersuchung von Ogilvie (1961) wertvoll.

36. Val Max. 4,1,10; Suet. Aug. 97,1.

37. S. dazu Demougin (1988), S. 217-225.

38. Das erlaubt uns, die Hypothese einer Anspielung auf den ludus Troiae auszuschließen.

39. Taylor (1931), S. 200-202. Doch die Frage bleibt sehr umstritten, vgl. Spannagel (1999), S. 24 f.

40. Vita Marci 6.

41. Suet. Aug.38,3.

42. Suet. ibid.; Cass. Dio 62,13,3 bezeichnet die transvectio zur Zeit Neros mit dem Wort $\dot{\varepsilon}^{\prime} \dot{\varepsilon} \tau \alpha \sigma l \varsigma$, das von Plutarch für die recognitio verwendet wird, während der sich Pompeius hervortut: Plut. Pomp. 22. S. Demougin (1988), S. 150-155.

43. S. dazu Mommsen (1887), III.1, S. 493-495; Ulp. Dig. 2,4,2: eum qui equo publico in Capitolium transvehatur.

44. S. dazu Mommsen (1887), II.1, S. 63.

45. ASC 72 St. Vgl. dazu Nicolet (1966), S. 327-330.

46. Diese große Bedeutung der neuen Apollon zugewiesenen Rolle wird häufig hervorgehoben; vgl. vor allem Sauron (1994), S. 526-527.

47. Suet. Caes. 44,2.

48. Taylor (1931), S. 200.

49. App. $B C 2,68$.

50. Cass. Dio. 43,23,4; vgl. Platner / Ashby (1929), S. 128.

51. Zu dieser Frage s. Gagé (1932), dem wir hier sehr verpflichtet sind.

52. Cass. Dio 54,8,3; s. dagegen Hor. c. 4,15,6.

53. S. dazu Cassola (1981), und zuletzt Simpson (1993), S. 116-122, mit der vorangehenden Bibliographie. Die Frage der Feier anlässlich der Rückkehr der parthischen Feldzeichen ist ausführlich aufgenommen worden von Rich (1998), S. 71-128, der eine neue Perspektive verfolgt und den Akzent auf den Unterschied zwischen den Augustus zugesprochenen Ehren und deren tatsächlicher Durchführung legt.

54. Dion. Halic. 2,34,4: Crawford, Roman Republican Coinage nr. 439. 
55. Cassola (1970), besonders S. 20-22.

56. Mattingly, BMC 1, Aug., nr. 704.

57. Ibid. nr. 315, 329, 366, 367, 369 bis 374,384 bis 389 .

58. Ibid. nr.397.

59. Alföldi (1952), (1953), (1954). S. auch Béranger (1953), S. 275-278.

60. Cass. Dio 55,5,1; im Jahre 29, Mon. Ancyr. 4.

61. Cass. Dio 55,1,1.

62. Cass. Dio 55,10,10.

63. Mon. Ancyr. $35,1$.

64. Mon. Ancyr. $29,2$.

65. Ov. fast. 5,565. Cassola (1970), S. 21, hat das bemerkt.

66. Ov. fast. 5,554 .

67. Zur umstrittenen Frage des Datums, 12. Mai oder 1. August, s. zuletzt Rich (1998) und Spannagel (1999), S. 41-59.

68. Suet. Aug. 30,4.

69. Mon. Ancyr. 20,1.

INDEX

Schlüsselwörter : Rom, Augustus, lex templi, Ideologie, Ritual Mots-clés : Rome, Auguste, lex templi, idéologie, rituel

\section{AUTEURS}

\section{MARIANNE BONNEFOND-COUDRY}

Marianne Bonnefond-Coudry ist emeritierte Professorin für Geschichte an den Universitäten Nancy und Mulhouse. Weitere Informationen finden Sie hier. 